\section{GENRE}

en séries

\section{Genre en séries}

Cinéma, télévision, médias

$11 \mid 2020$

Freaks en tous genres : corps mutants, cyborgs, métamorphoses \& fantastiques

\title{
Marianne Kac-Vergne, Masculinity in Contemporary Science Fiction Cinema. Cyborgs, Troopers and Other Men of the Future
}

London, I.B.Tauris, 2018, 246 pages

Michel Bondurand Mouawad

\section{(2) OpenEdition}

\section{Journals}

Édition électronique

URL : http://journals.openedition.org/ges/1091

DOI : 10.4000/ges.1091

ISSN : 2437-6563

Éditeur

Presses universitaires de Bordeaux

Référence électronique

Michel Bondurand Mouawad, « Marianne Kac-Vergne, Masculinity in Contemporary Science Fiction Cinema. Cyborgs, Troopers and Other Men of the Future », Genre en séries [En ligne], 11 | 2020, mis en ligne le 01 juin 2020, consulté le 18 février 2021. URL : http://journals.openedition.org/ges/1091 ; DOI : https://doi.org/10.4000/ges.1091

Ce document a été généré automatiquement le 18 février 2021.

\section{C)}

La revue Genre en séries est mise à disposition selon les termes de la Licence Creative Commons Attribution - Pas d'Utilisation Commerciale - Pas de Modification 4.0 International. 


\section{Marianne Kac-Vergne, Masculinity in Contemporary Science Fiction Cinema. Cyborgs, Troopers and Other Men of the} Future

London, I.B.Tauris, 2018, 246 pages

Michel Bondurand Mouawad

\section{RÉFÉRENCE}

Marianne Kac-Vergne, Masculinity in Contemporary Science Fiction Cinema. Cyborgs, Troopers and Other Men of the Future, London, I.B.Tauris, 2018, 246 pages

1 Marianne Kac-Vergne, maîtresse de conférences en civilisation américaine à l'université de Picardie Jules Verne, publie en anglais un magnifique ouvrage consacré à l'analyse culturelle des masculinités dans le cinéma de science-fiction hollywoodien. Son corpus rassemble principalement des films produits entre les années 1980 et la fin des années 2000 - bien qu'elle fasse judicieusement quelques comparaisons avec des productions antérieures à cette période.

2 Sa démarche part d'un constat simple: le manque d'intérêt dans la littérature consacrée à la science-fiction pour les dimensions genrées des représentations, qui s'accompagne notamment d'une confusion, implicite mais centrale, entre masculinité et humanité, plus visible encore dans les termes anglais de "man » et «mankind». L'autrice élabore sa méthode dans l'héritage du travail fondamental de R. W. Connell sur les masculinités ${ }^{1}$ et, dans son sillon, prête une attention particulière à la manière dont la masculinité hégémonique est redéfinie à l'écran par opposition aux femmes, mais aussi aux représentants des masculinités subalternes. Pour cela, Kac-Vergne étudie méticuleusement son corpus dans une perspective intersectionnelle qui articule l'analyse des identités et rapports de genre, de sexualité, de race, de classe sociale et de 
nationalité. L'ouvrage est organisé en cinq chapitres thématiques précédés d'une courte introduction méthodologique et conclus par une brève synthèse.

\section{Les années 1980 et les paradoxes d'une « hypermasculinité vulnérable »}

3 Le premier chapitre met en lumière un apparent paradoxe au cœur des productions de science-fiction des années 1980: leur représentation d'une «hypermasculinité vulnérable». L'autrice rappelle avec pertinence le contexte socioculturel de la présidence Reagan et les travaux qui décrivent ces années comme une période de «backlash» contre l'avancée des droits des minorités lors des deux décennies précédentes. Elle rappelle fort à propos les écrits désormais classiques de Susan Jeffords et d'Yvonne Tasker sur la «remasculinisation de l'Amérique » à travers les corps bodybuildés «spectaculaires" des stars masculines de l'époque telles Arnorld Schwarzenegger et Sylvester Stallone ${ }^{2}$. Mais Kac-Vergne voit apparaître un paradoxe dans le genre de la science-fiction, à travers la présence de héros masculins dont le corps apparaît comme pénétrable, c'est-à-dire faillible et vulnérable, et qui sont présentés comme des victimes nées d'un traumatisme. L'autrice remarque ainsi que ce portrait d'une masculinité vulnérable cohabite avec de nombreuses scènes où le corps hypermasculin est exhibé totalement nu d'une manière qui insiste sur sa puissance "naturelle» (une qualité qui semble alors réservée aux corps blancs, tandis que les non-blancs forment la masse d'antagonistes joyeusement éliminés au cours des films). Kac-Vergne souligne ainsi la tension interne de ces films structurés autour du paradoxe de corps masculins puissants mais faillibles et constamment en proie à la pénétration et à la destruction, comme l'illustrent les nombreuses scènes de torture qui transforment le corps blanc masculin en territoire de souffrance et de trauma propre à la sympathie et au soutien du public.

4 L'étude montre également que le genre science-fictionnel ne se réduit pas à une simple apologie de l'hypermasculinité et que ces productions remettent en cause ces masculinités données comme «naturellement » puissantes et dominantes à travers les nombreux récits qui insistent sur l'hybridité des corps humains/machines et l'instabilité des signes d'humanité. Les travaux classiques de Donna Haraway sur la figure du cyborg ont montré le potentiel révolutionnaire de ces créatures qui remettent en question la fixité des marqueurs identitaires ${ }^{3}$. Mais au-delà de cette critique de niche, le corps hypermasculin assimilé à une machine sert également à inquiéter et alarmer le public sur les risques de déshumanisation inhérents à une époque où les progrès de la robotisation bouleversent le secteur industriel et toute l'économie américaine déjà chamboulée par les mesures ultralibérales prises par l'administration Reagan.

\section{Dystopies et héroïsme hypermasculin des classes populaires blanches des années 80}

5 Le deuxième chapitre, "Dystopie et guerre de classe ", est consacré à la présence de nouvelles fractures de classe à l'intérieur des récits dystopiques des années 1980. KacVergne rappelle très justement que la critique américaine est elle-même peu sensible à 
la représentation des identités de classe dans les films. Le mythe national d'une société sans classes est encore prégnant et cette dimension est rarement perçue par les publics, critiques comme spectateurs/trices ordinaires. Or ce chapitre cerne, dans les dystopies de l'époque, une critique des élites économiques et financières à travers la personnification d'antagonistes clairement représentants de l'idéologie "greed is good» ("la cupidité est une bonne chose ») devenue le mot d'ordre de la génération yuppie née de la dérèglementation des marchés organisée par les ultralibéraux au cours de la décennie. L'avenir n'est pas rose dans ces récits futuristes : la vie urbaine est montrée comme décadente et dangereuse, la peur de l'invasion est omniprésente et les héros sont des marginaux. La vision du futur qui domine les écrans est traversée par des angoisses typiques de l'époque Reagan, notamment une technophobie qui voit le progrès technologique comme la source principale d'une déshumanisation de la société. Les angoisses liées à la robotisation des industries, aux privatisations sauvages dans tous les secteurs, à l'injonction à la richesse et la culpabilisation des déclassé.e.s, se trouvent incarnés dans des mondes angoissants dépeints comme hyper-compétitifs et hyper-hiérarchisés. Des films majeurs comme Blade Runner (Ridley Scott, 1982), RoboCop (Paul Verhoeven, 1987) ou Total Recall (Paul Verhoeven, 1990) mettent en scène une guerre de classe entre des héros issus de classes populaires et des antagonistes appartenant à l'élite socio-économique.

6 Il existe cependant une forte ambivalence dans la représentation de celles et ceux considéré.e.s comme des déclassé.e.s. Souvent assimilé.e.s à des mutant.e.s, les plus défavorisé.e.s rappellent les populations non blanches qui peuplent les centres-villes des métropoles états-uniennes, perçues à la fois comme criminelles et opprimées. À l'époque, plusieurs révoltes en cours - comme celles de l'IRA en Irlande ou de l'ANC en Afrique du Sud - rappellent aux foyers américains que les terroristes des uns sont les héros des autres. Or les héros des films de science-fiction sont blancs et viennent systématiquement à bout des hordes de mutants comme pour exorciser le déclassement des classes populaires américaines, perçu comme la conséquence d'une prise de pouvoir des minorités, femmes et non-blancs. La dystopie joue ainsi un rôle symbolique de compensation en célébrant l'héroïsme hypermasculin des classes populaires blanches.

7 L'autrice montre parfaitement toute la subtilité des dystopies des années 1980 . Celles-ci peuvent être vues en partie comme une critique satirique des dérèglements socioéconomiques provoqués par les années Reagan qui amorcent un long processus de déclassement pour les classes populaires blanches. Mais ces films sont aussi fort habiles à utiliser les codes du genre afin de célébrer et de ritualiser l'incorporation de ces mêmes strates sociales à l'intérieur d'un bien commun et apaiser ainsi symboliquement les tensions de classes.

\section{Des femmes en marge}

8 Le troisième chapitre explore les procédés de « Marginalisation des femmes » dans les films du corpus. Kac-Vergne calcule que sur les 130 productions majeures du genre, seules 18 comportent un personnage principal féminin, ce qui équivaut à moins de $14 \%$ de la production entre 1980 et 2015. Même si la fin du vingtième siècle a vu le remplacement des acolytes masculins par des acolytes féminines dotées d'une nouvelle capacité d'agir et jouant un rôle de médiatrices du regard spectatoriel, ces dernières 
servent principalement à marquer la différence sexuelle et à garantir l'hétéronormativité du récit. Les années 1980 avaient toutefois vu naître de véritables héroïnes de science-fiction, notamment les célèbres Ripley (franchise Alien) et Sarah Connors (Terminator 1 \& 2). Ripley incarne parfaitement les changements sociétaux des deux décennies précédentes, notamment ceux issus de la deuxième vague du féminisme. La neutralité de genre qui avait informé la création du personnage laisse place, au fil des épisodes suivants, à sa masculinisation - à travers son développement musculaire et son goût pour les armes - ainsi qu'à un nouvel accent mis sur la maternité mais aussi sur l'émotion et le trauma pour ce personnage rendu fragile, en proie à un sentiment d'incomplétude. James Cameron, qui avait déjà transformé Ripley dans son Aliens (1986), use des mêmes tactiques pour faire passer Sarah Connors de simple acolyte du héros masculin dans Terminator (1984) au statut d'héroïne d'action musclée et armée jusqu'aux dents dans Terminator 2 (1991). Même si la masculinisation d'une part, et la stigmatisation par la maternité et l'instabilité émotionnelle d'autre part, semblent être un passage obligé pour accéder au statut d'héroïne, il est indéniable que ces deux personnages ont marqué la décennie et n'ont guère eu de descendance.

9 En effet, Kac-Vergne remarque qu'au cours des années 1990, les femmes retombent rapidement au second plan des récits. Son analyse de Starship Troopers (Paul Verhoeven, 1997) et de Ghosts of Mars (John Carpenter, 2001) montre comment les années 1990, puis 2000, vont abandonner la construction de personnages féminins issus des revendications d'égalité et de partage du pouvoir au profit d'une tendance au glamour et à l'hypersexualisation de femmes visibles mais renvoyés au second plan. L'autrice considère que l'idéologie post-féministe, qui caractérise le tournant du XXI ${ }^{\mathrm{e}}$ siècle, est responsable de cette mise à l'écart de l'héroïsme féminin, et porte son attention sur quelques figures typiques qui peuplent désormais les films de science-fiction, notamment celle de la «dominatrice » (la méchante de Terminator 3 par exemple) ou de la «babe scientist» (poupée savante). Les personnages féminins de notre époque semblent voués à servir et à valoriser une position héroïque qui leur échappe dorénavant.

\section{Une arrivée ambivalente de personnages noirs dans le genre}

10 Le quatrième chapitre est consacré à l'étude des personnages noirs. L'autrice rappelle l'absence de ces personnages dans l'histoire du genre jusqu'aux années 1990. Cette décennie suit une période de repli sur des valeurs conservatrices en réponse à l'ouverture des droits civiques et à l'intégration des noir.e.s dans la société étatsunienne. La période est meurtrie par de violentes émeutes raciales dont la plus célèbre reste celle de 1992 à Los Angeles au lendemain de la diffusion des images du meurtre de Rodney King par des agents de la LAPD. Dans le même temps, la démographie noire évolue et de nombreux ménages sortent d'une pauvreté endémique pour former une nouvelle classe moyenne. Le chapitre s'intéresse à la manière dont le cinéma de science-fiction intègre de nouveaux personnages noirs aux récits afin de toucher de nouveaux publics, tout en perpétuant certains stéréotypes raciaux qui structurent l'imaginaire états-unien. Une question centrale est celle de savoir s'il est alors possible pour un personnage noir de représenter l'humanité dans le cadre de ce genre historiquement dominé par les blancs. 
11 Kac-Vergne analyse en détail Predator 2 (Stephen Hopkins, 1990) et Demolition Man (Marco Brambilla, 1993) qui mettent en avant deux protagonistes noirs incarnés respectivement par Danny Glover et Wesley Snipes. Si leur masculinité impulsive et belliqueuse se place spontanément dans l'héritage du stéréotype du noir sauvage (le «black buck »), les deux films utilisent leur personnage racisé afin de jeter une lumière critique sur la faillite de l'ordre blanc traditionnel, et légitiment dans une certaine mesure la violence des personnages noirs à travers la peinture d'élites blanches incapables ou corrompues. En soulignant les difficultés propres aux protagonistes nonblancs qui doivent faire face à des animosités particulières en provenance de leur entourage, ces films dénoncent de surcroît le racisme quotidien que subissent les Africains-Américains.

Le film Independence Day (Roland Emmerich, 1996) introduit l'analyse fouillée que KacVergne réserve au vedettariat de Will Smith. Dans ce film, la masculinité de Smith est traitée comme dans la décennie précédente : son corps musclé est exhibé et mis en valeur, son héroïsme met à l'écart les protagonistes féminins, et son succès valide le système patriarcal qu'il rétablit en ramenant l'ordre dans le monde. Cependant, le personnage joué par Will Smith reste ici au service d'un président blanc avec qui il partage la lumière des projecteurs. D'autres films analysés - notamment la franchise Matrix - montrent que la période ne remet pas fondamentalement en cause la primauté d'un ordre blanc même si une nouvelle place est faite aux personnages racisés.

L'autrice conclut le chapitre en montrant comment les personnages noirs ont remis en question l'hégémonie blanche sans vraiment fragiliser la domination masculine, mais en l'intégrant avec plus ou moins d'aisance.

\section{Les années 2000 ou le retour d'un patriarcat triomphant}

14 Le dernier chapitre est justement consacré au retour de l'hégémonie masculine au lendemain des attaques du 11 septembre et des guerres au Moyen-Orient. Kac-Vergne montre clairement comment le discours d'une crise de la masculinité sous Clinton a préparé le retour de récits autour du rétablissement d'un patriarcat fort et puissant au lendemain des attaques sur New York et sur Washington. En effet, quelques films sous Clinton - notamment réalisés par des femmes cis (Strange Days, Kathryn Bigelow, 1995) et trans (la franchise Matrix par les sœurs Wachowski) - avaient proposé un héroïsme masculin largement adouci et débarrassé de quelques prérogatives. La douce virilité de Keanu Reeves s'incarne souvent dans des personnages qui s'éloignent des modèles hypermasculins des années 1980. Caractérisés par une certaine passivité, ils fuient les représentants de l'hégémonie masculine traditionnelle pour chercher refuge et assistance auprès de protagonistes féminines qui paraissent fortes et puissantes. Dans ces films, les femmes semblent combler le vide politique et corporel laissé par la passivité des corps masculins blancs. Elles forcent les hommes à rester dans la réalité alors que ces derniers sont tentés par le virtuel. L'autrice remarque d'ailleurs que ces protagonistes portent le désir et engagent les héros masculins dans des relations charnelles qui disparaitront des écrans dans la décennie suivante.

Les années 2000 sont celles du retour d'un patriarcat triomphant. La science-fiction était jusqu'ici peu encline à l'éloge de la famille qui s'était déjà imposé dans d'autres 
genres, notamment celui de l'action et de l'aventure. Au lendemain des attaques sur le sol américain, les récits apocalyptiques fleurissent et exhortent les pères à prendre soin de leur progéniture dans un monde insensé. Ces récits mettent en parallèle l'histoire d'une catastrophe et celle d'une crise familiale, à l'image de War of the Worlds (Steven Spielberg, 2005), et permettent ainsi de légitimer à bon compte la prise du pouvoir du père au niveau micro- et macro-sociétal. L'omnipotence du patriarcat est également justifiée par une idéologie post-féministe qui confie aux hommes le soin et l'empathie émotionnelle. Une nouvelle masculinité hégémonique est ainsi refondée grâce à la mise en avant d'aptitudes physiques exceptionnelles, du maniement obligatoire des armes dont l'utilisation est légitimée par de nombreuses scènes qui organisent une menace sur leur progéniture - mais aussi de la prise en charge des tâches relatives au soin et à l'empathie, facilitant ainsi l'expulsion des personnages féminins hors du récit.

Pourtant, comme si une conscience autoréférentielle régnait dans les films de la période, l'hégémonie masculine et le patriarcat sont mis à mal et soumis à des moments d'échec. La progéniture se rebelle presque systématiquement et refuse de suivre la route tracée par le père. Les héros sont raillés et leur idéalisme passe pour futile. Dans une époque postmoderne où l'ironie est le mode d'expression privilégié, l'héroïsme masculin se doit d'avancer masqué. Kac-Vergne remarque d'ailleurs que les récits mettent plus volontiers en avant les relations père-fille qui permettent selon elle de ramener du féminin dans un champ monopolisé par la masculinité.

On ne peut s'empêcher de regretter que ce précieux travail ne soit pas disponible en français. Marianne Kac-Vergne livre une analyse très équilibrée entre contextualisation socioculturelle et analyse filmique. Deux remarques pourraient être faites pour compléter cette lecture. On peut s'étonner que la riche intersectionnalité utilisée par l'autrice tout au long de l'ouvrage n'ait pas mieux éclairé les sexualités des masculinités décrites. La dimension hétérosexuelle se retrouve souvent mentionnée, mais il aurait été intéressant d'approfondir l'analyse de ces représentations, notamment en termes de filiation, de fertilité et de reproduction, trois thèmes qui sont devenus majeurs dans la société états-unienne des années 2000. Enfin, il devient urgent pour les spécialistes des constructions identitaires d'inclure la dimension spécielle dans ces analyses. La masculinité est également formée par notre identité en tant qu'espèce et elle joue un rôle majeur dans le rapport que nos cultures entretiennent avec le monde naturel, et notamment avec les animaux. Les films de science-fiction qui imaginent la fin d'un monde désormais annoncée dans la réalité, se prêteraient tout particulièrement à cette analyse, comme ceux qui traitent du rapport entre l'espèce humaine et d'autres espèces animales, à l'image de la franchise La Planète des singes.

\section{NOTES}

1. R.W. Connell, Masculinities, Sydney, Allen \& Unwin, 1995. Partiellement traduit en français et disponible sous le titre Masculinités : Enjeux sociaux de l'hégémonie, Paris, Amsterdam, 2014. 
2. Susan Jeffords, The Remasculinization of America: Gender and the Vietnam War, Indiana University Press, 1989 ; Yvonne Tasker, Spectacular Bodies: Gender, Genre and the Action Cinema, New York, Routledge, 1993.

3. Donna Haraway, Simians, Cyborgs, and Women: The Reinvention of Nature, New York, Routledge, 1991.

\section{AUTEURS}

\section{MICHEL BONDURAND MOUAWAD}

Michel Bondurand Mouawad est docteur en études cinématographiques, il dirige les centres des Universités de la Caroline du Nord et de l'Illinois en France. Il est également chargé de cours à l'université Paris 3 Sorbonne Nouvelle. Ses dernières publications portent sur l'analyse culturelle des représentations de la violence politique dans les productions hollywoodiennes contemporaines. Sur ce sujet, La Chair de mon ennemi : une histoire sexuelle du terrorisme hollywoodien paraîtra prochainement aux éditions du Murmure. 